\title{
El vínculo educación y trabajo desde la perspectiva de estudiantes secundarios en Caleta Olivia (Santa Cruz)
}

\section{The link between education and job considering the perspective of students of high school in Caleta Olivia (Santa Cruz)}

\author{
Mauro Victor Guzmán ${ }^{1}$ \\ Silvia Mariela Grinberg ${ }^{2}$ \\ Eduardo Daniel Langer ${ }^{3}$
}

\section{Resumen}

Este artículo propone una exploración del vínculo educación y trabajo considerando las perspectivas de estudiantes del Nivel Secundario en la localidad de Caleta Olivia (Santa Cruz, Argentina). A partir de datos de una encuesta realizada en 2018, se indaga en sus considerandos sobre la importancia de la escuela y las razones por las que asisten. Ello se analiza en relación con el emplazamiento urbano de las instituciones, esto es, atendiendo a la desigualdad socioeducativa (según NBI de radio censal), la modalidad de la escuela y el año en curso. Los resultados de las encuestas realizadas expresan que, si bien la totalidad de estudiantes acuerdan sobre la relevancia de la escuela para el trabajo, se relativiza aquella centralidad al indagar sobre sus escolaridades y la relación con las posibilidades de empleo. Ello a partir de diferenciaciones que se producen entre quienes concurren a escuelas emplazadas en las áreas más empobrecidas de la ciudad, aquellos que transitan el último año escolar, así como en función de la orientación que cursan.

Palabras clave: educación secundaria; trabajo; estudiantes.

\section{Abstratc}

This article proposes an exploration of the link education and work considering the perspectives of students of the secondary level in the town of Caleta Olivia (Santa Cruz,

\footnotetext{
${ }^{1}$ CONICET-UNPA. Correo: viktorm_g@hotmail.com

${ }^{2}$ CONICET-UNSAM-UNPA. Correo: grinberg.silvia@gmail.com

${ }^{3}$ CONICET-UNSAM-UNPA. Correo: langereduardo@gmail.com
} 
Argentina). Based on data from a 2018 survey, your considerations about importance of the school and the reasons why they attend are analyzed. This is done in relation to the urban location of the institutions, taking into account socio-educational inequality (according to the census radio $\mathrm{NBI}$ ), the type of school and the current year. The results of the surveys carried out express that although all students agree on the relevance of the school for work, this centrality is relativized when inquiring about their schooling and the relationship with employment possibilities. This is based on differentiations that occur between those who attend schools located in the most impoverished areas of the city, those who go through the last school year as well as depending on the orientation they attend.

Keyword: high school, job, students.

\section{Introducción}

Desde fines del siglo $\mathrm{XX}$, a partir de las mutaciones del proceso productivo en el capitalismo (Castel, 1997; Harvey, 2000; Antunez, 2009), han cobrado centralidad los debates respecto a las utilidades que la formación escolar tiene con relación al trabajo. Diversos estudios se han centrado en la ruptura de las linealidades que caracterizaban el pasaje de la escuela al trabajo en tiempos fordista-keynesianos (Dubar, 2001, Gallart, 1997; Jacinto, 2010). Ello ha implicado desde fines de los 90 el desarrollo de estudios que han analizado el problema de la inserción (Dubar, 2001) o la transición de la escuela al trabajo (Jacinto, 2010; Miranda, 2017). La pregunta por la formación escolar implica situarnos en un contexto en que, como sostiene Deleuze (2006), nada se termina nunca. Es decir, la formación permanente, el aprender a aprender, aquello que va a definir la empleabilidad de los sujetos (Gallart, 1997) forma parte de las lógicas de gobierno de las conductas en las sociedades empresa (Foucault, 2007). Centramos nuestra mirada en el golfo San Jorge, una región en que las dinámicas globales del mercado de trabajo adquieren ciertas particularidades asociadas a los ritmos y lógicas flexibles del capital transnacional. Estas producen efectos en las dinámicas 
sociolaborales locales, dado que históricamente han estado y aún están condicionadas por las actividades extractivas de petróleo (Schweitzer, 2012; Márquez, 2017).

En este marco de debates, nos preguntamos por la relación entre educación y trabajo, recuperando las perspectivas de estudiantes de Nivel Secundario que asisten a escuelas públicas de Caleta Olivia (provincia de Santa Cruz, Argentina) ${ }^{4}$. Se propone caracterizar sus considerandos sobre la importancia de la escuela con relación al trabajo, teniendo en cuenta las características del mercado laboral en esta ciudad intermedia ${ }^{5}$ (Bellet y Llop, 2004), las dinámicas de las escuelas a las que asisten los y las estudiantes y el año de cursada en que se encuentran. Para ello, uno de los ejes centrales será el de las desigualdades sociales en función de las condiciones de emplazamiento urbano de las escuelas, así como las desigualdades escolares en función de la modalidad de la formación ofrecida. Ello por las diferencias históricas en el sistema educativo argentino y en particular, en la región del golfo San Jorge, entre la formación técnica, más estrechamente vinculada al trabajo, y la formación común o de bachillerato.

En el artículo se trabajan resultados de investigación a través de una propuesta metodológica de base múltiple, partiendo de una combinación entre datos cualitativos y cuantitativos de información. La indagación sobre la perspectiva de estudiantes en relación con el emplazamiento de las escuelas se realiza a partir de la combinación de dos tipos de datos que se georreferenciaron en el espacio urbano: I) Encuestas a estudiantes respecto a la importancia escolar y motivaciones en sus escolaridades. Las encuestas se efectuaron sobre la base de una muestra intencional y criterios de selección atendiendo al: emplazamiento urbano de las instituciones, índices de rendimiento escolar ${ }^{6}$ y las orientaciones de la escolaridad secundaria. II) Datos sociodemográficos del emplazamiento de las escuelas a

\footnotetext{
${ }^{4}$ Se trabaja con resultados de investigación en el marco de una beca doctoral otorgada por CONICET, cuyo plan de tesis se denomina "Formación para el trabajo y espacio urbano fragmentado: un estudio en escuelas secundarias de la región del golfo San Jorge".

${ }^{5}$ Siguiendo a estos autores, consideramos que Caleta Olivia es una ciudad intermedia, en la medida en que se concentran actividades y servicios especializados, las cuales sirven de "intermediación" e integración entre procesos locales, nacionales e internacionales.

${ }^{6}$ Se tomó para ello los indicadores de repitencia, no promoción y sobreedad del ciclo lectivo 2014, último año con información disponible otorgada por el Consejo Provincial de Educación de Santa Cruz.
} 
partir de índices de Necesidades Básicas Insatisfechas $\left(\mathrm{NBI}^{7}\right)$ obtenidos del último Censo nacional del Indec en 2010.

Para la muestra de bachilleratos, en primer lugar, se georreferenciaron los índices de NBI del radio censal en que se emplazan las escuelas mediante el uso de Sistemas de Información Geográfica (SIG). Como cada uno de los radios censales está compuesto por un porcentaje de hogares con $\mathrm{NBI}$, aquí se han organizado tres terciles, cuyos polos van desde el "NBI Bajo" (radios con menor cantidad de hogares con pobreza) al "NBI Alto" (radios con mayor cantidad de hogares en situación de pobreza). A partir de allí se han seleccionado escuelas de bachillerato según el emplazamiento en cada tercil de $\mathrm{NBI}^{8}$, a la vez que se tuvo en cuenta el comportamiento de los indicadores educativos y la oferta de orientaciones procurando que estas sean diversas. En cuanto a las escuelas técnicas, se han seleccionado las dos que en Caleta Olivia tienen distintas orientaciones y que en 2018 ofrecían los seis años que contempla el trayecto formativo.

Tal como expresa el Mapa $\mathrm{N}^{\circ}$ 1, en el tercil NBI Bajo hay una institución educativa que corresponde al segundo bachillerato público más antiguo de Caleta Olivia (creado en 1977) y uno de los más demandados de la localidad en épocas de inscripciones, así como una de las instituciones que más rápidamente queda sin vacante. En esta institución se ofrecen dos orientaciones: Humanidades y Ciencias Sociales y Turismo (Escuela 1). En el NBI Medio hay una institución educativa que corresponde al bachillerato público más antiguo de la localidad (creado en 1962) y ofrece dos orientaciones: Humanidades y Ciencias Sociales y Economía y Administración (Escuela 2). En el tercil NBI Alto hay dos instituciones educativas, ambas fueron creadas en 2013 y corresponden a los últimos bachilleratos públicos que se crearon en la localidad. Las dos escuelas ofrecen solo una orientación: una de ellas ofrece la orientación de Artes Visuales (Escuela 4) y la otra tiene la orientación de Ciencias Sociales (Escuela 3). Por último, para la comparación por modalidades, consideramos dos escuelas

\footnotetext{
7 Dicho indicador es un método directo para identificar carencias críticas en una población y caracterizar la pobreza, que usualmente utiliza indicadores directamente relacionados con cuatro áreas de necesidades básicas de las personas (vivienda, servicios sanitarios, educación básica e ingreso mínimo), información que se obtiene de los censos de población y vivienda (Feres y Mancero, 2001).

${ }^{8}$ Por tanto, la significatividad de la relación entre NBI y tipo de escuela se produce en tanto que esa selección de la muestra fue intencional en función del emplazamiento urbano.
} 
técnicas. Una de ellas es de modalidad técnico industrial, creada en 1960, y es una de las instituciones que rápidamente queda sin vacante. Dicha institución ofrece cuatro orientaciones: Maestro Mayor de Obras, Industria de Procesos, Informática y Electromecánica (Escuela 5). La otra escuela técnica fue creada en 1993 y ofrece solo la orientación en Biología Marítima, Pesquería y Laboratorista (Escuela 6).

\section{Mapa 1. Ubicación de las escuelas secundarias seleccionadas según nivel de NBI del radio censal, Caleta Olivia, 2018}

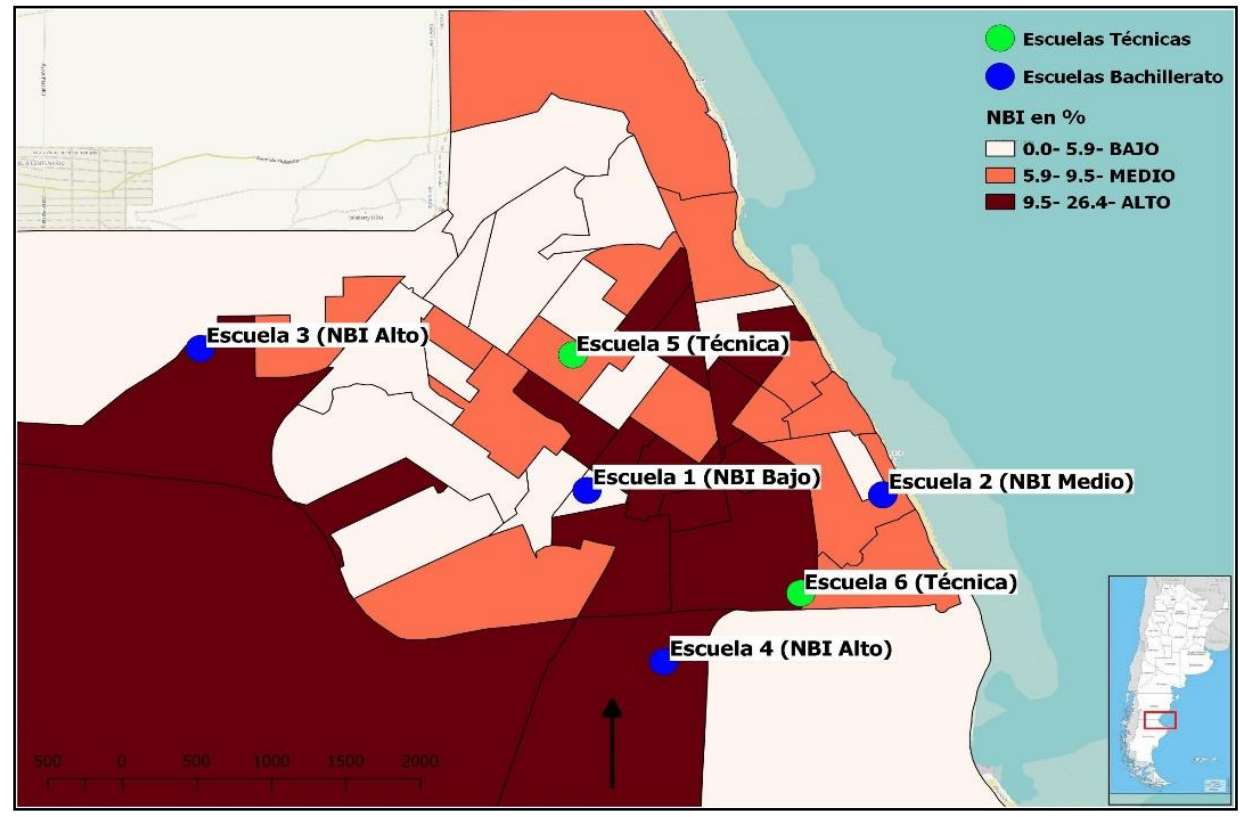

Fuente: elaboración propia, sobre la base de datos del Centro de Intercambio y Reservorio de Información Social y Educativo (CIRISE), alojado en el Área Sociopedagógica de la UNPAUACO.

Dentro de cada institución se eligieron al azar un curso del $1^{\circ}$ año del Nivel Secundario, un curso del $1^{\circ}$ año del Ciclo Orientado ( $3^{\circ}$ año de la secundaria) y un curso del último año del Ciclo Orientado ( $5^{\circ}$ año de bachilleratos y $6^{\circ}$ año de escuelas técnicas). Con este criterio se buscó abarcar la mirada de los y las estudiantes de todo el nivel, considerando los momentos clave relacionados al inicio del Nivel Secundario en general, el inicio del ciclo orientado y la perspectiva de quienes están a punto de finalizar la secundaria y "salir" hacia el mercado de trabajo. El instrumento de encuesta estuvo compuesto por 42 preguntas cerradas y abiertas, agrupadas en distintas dimensiones de análisis referidas a: información 
sociodemográfica, participación en espacios extraescolares, acceso y uso de TIC, motivaciones de asistencia escolar, actividades y saberes escolares, perspectivas a futuro. El cuestionario se implementó durante 2018 y se realizó de manera autoadministrada durante el horario escolar dispuesto por cada institución, con la presencia de al menos dos integrantes del equipo de investigación ${ }^{9}$. Su construcción fue realizada desde el equipo en interacción con participación activa de miembros de la comunidad escolar -supervisores, directores y docentes de las escuelas-, así como se han validado en pruebas piloto con estudiantes realizadas con anticipación a su aplicación. La sistematización y posterior análisis de la información relevada se realizó mediante la utilización del programa informático SPSS, el cual permite sistematizar el volumen de información cualitativa y cuantitativa de las encuestas realizadas sin mecanizar el proceso de análisis.

El relevamiento implicó un alcance de 487 estudiantes, pero al objeto de este trabajo se ha seleccionado la perspectiva de estudiantes del primer y último año del nivel, con lo que se trabaja con una muestra de 283 estudiantes.

Tabla $\mathbf{N}^{\circ}$ 1. Descripción de la muestra de estudiantes. En \%. Caleta Olivia. $(\mathbf{N}=\mathbf{2 8 3})$

\begin{tabular}{|l|l|r|r|}
\hline \multirow{2}{*}{} & \multicolumn{2}{|c|}{ CURSO ESCOLAR } \\
\cline { 3 - 4 } & & Primer Año & Último Año \\
\hline \multirow{4}{*}{ GÉNERO } & Masculino & 49,6 & 45,9 \\
\cline { 2 - 4 } & Femenino & 48,8 & 53,0 \\
\cline { 2 - 4 } & No responde & 1,7 & 1,1 \\
\hline \multirow{5}{*}{ Total } & 100 & 100 \\
\hline \multirow{5}{*}{ EDAD } & 12 años & 10,7 & \\
\cline { 2 - 4 } & 13 años & 61,2 & \\
\cline { 2 - 4 } & 14 años & 19,0 & \\
\cline { 2 - 4 } & 15 años & 5,0 & \\
\cline { 2 - 4 } & 16 años & 0,8 & 3,9 \\
\cline { 2 - 4 } & 17 años & 1,7 & 23,8 \\
\cline { 2 - 4 } & 18 años & 0,8 & 43,6 \\
\cline { 2 - 4 } & 19 años & & 18,8 \\
\cline { 2 - 4 } & 20 años & & 5,0 \\
\cline { 2 - 4 } & 21 años & & 1,7 \\
\cline { 2 - 4 } & & &
\end{tabular}

\footnotetext{
${ }^{9}$ Se trata de una encuesta desarrollada en el marco del PDTS UNPA-Conicet, Centro de Intercambio y Reservorio de Información Social y Educativo (CIRISE). Disponible en: http://www.uaco.unpa.edu.ar:8533/cirise/
} 


\begin{tabular}{|l|l|r|r|}
\hline \multicolumn{2}{|c|}{} & \multicolumn{2}{|c|}{ CURSO ESCOLAR } \\
\cline { 3 - 4 } \multicolumn{2}{|c|}{} & Primer Año & Último Año \\
\hline \multirow{3}{*}{ GÉNERO } & Masculino & 49,6 & 45,9 \\
\cline { 2 - 4 } & Femenino & 48,8 & 53,0 \\
\cline { 2 - 4 } & No responde & 1,7 & 1,1 \\
\cline { 2 - 4 } & 22 años & & 0,6 \\
\cline { 2 - 4 } & No responde & $0,8 \%$ & 2,2 \\
\hline \multirow{2}{*}{ Total } & & 100 & 100 \\
\hline
\end{tabular}

Fuente: elaboración propia.

Tal como se observa en la Tabla $N^{\circ} 1$, la encuesta se ha realizado a estudiantes de: a) primer año en el que la distribución por género fue semejante (el 49,6\% varones y el 48,8\% mujeres) y b) del último año fue levemente mayor hacia la proporción de mujeres (53\%) por sobre la de varones (45,9\%). Asimismo, las edades de los estudiantes de primer año son principalmente entre 12 y 14 años, mientras que en último año la edad se concentra en el rango de 17 a 19 años.

Estructuramos el siguiente trabajo en tres partes. En primer lugar, abordamos las características que asumen las relaciones entre educación y trabajo, atendiendo a la configuración de la organización del trabajo y saberes para el trabajo en tiempos de capitalismo flexible. Ello en un escenario sociolaboral de una ciudad intermedia como Caleta Olivia, donde las reconfiguraciones propias de fines del siglo XX dejaron en crisis a la actividad petrolera. Nos referimos a una ciudad cuya actividad principal sigue asociada a la industria del petróleo, pero atravesada por los constantes vaivenes y flujos globales. Luego, se describen las miradas que estudiantes de escuelas secundarias de la localidad tienen sobre la importancia de la escuela en general, sus razones para asistir a la institución particular a la que van y cómo estas motivaciones se relacionan con el trabajo. Estas lecturas tendrán en cuenta el emplazamiento urbano de las escuelas y si son de modalidad técnica o bachillerato. Finalizamos con reflexiones que dan cuenta de las características que asumen la formación para el trabajo desde la escuela secundaria, cómo se relacionan con las características sociourbanas de emplazamiento escolar y la modalidad de las instituciones. 


\section{Mutaciones en el mundo laboral y la formación para el trabajo}

Desde hace casi medio siglo se han vivido procesos de cambios profundos con relación a los modos de producción y circulación de la riqueza bajo lógicas flexibles (Harvey, 2000; Sennett, 2000). Diversos estudios han señalado que el proceso de precarización del trabajo devenido de la crisis de la sociedad salarial (Castel, 1997) ha tenido características particulares en Latinoamérica, ello dado la heterogeneidad productiva que la caracteriza (De la Garza, 2000; Antunes, 2009; Jacinto, 2013; De Ibarrola, 2016). En este sentido, cabe considerar que Caleta Olivia no ha estado ajena a los procesos de precarización del trabajo, ya que la estructura productiva asociada históricamente a la extracción de petróleo presenta las características de los ritmos y lógicas flexibles del capital transnacional (Schweitzer, 2012). Caleta Olivia, al igual que las demás localidades que conforman el golfo San Jorge ${ }^{10}$, se ha desarrollado bajo una lógica de enclave petrolero (Salvia, 1997). Es decir, no solo nos referimos al desarrollo económico de la mano de la empresa estatal Yacimientos Petrolíferos Fiscales $(\mathrm{YPF})^{11}$, sino también al conjunto de relaciones sociales asalariadas que se configuraron en las localidades de la región (Márquez, 1995; Salvia, 1997; Ruiz y Muñoz, 2008). De allí que la privatización de YPF y la reconfiguración del proceso productivo bajo las lógicas flexibles del capital a fines del siglo $\mathrm{XX}$, produjeron efectos desestructurantes en el mercado de trabajo y los trabajadores (Salvia, 1997; Ruiz y Muñoz, 2008). Aunque coyunturalmente hubo períodos de mejoramiento y crisis en los últimos años, estructuralmente esos efectos aún persisten en las luchas que se desarrollan para mejorar las condiciones laborales o evitar el desempleo (Schweitzer, 2012; Márquez, 2017). De hecho, Schweitzer (2012) señala que las dinámicas sociolaborales de la región del golfo San Jorge configuran sociedades polarizadas ya que se componen por sectores con salarios muy altos que se vinculan a la actividad extractiva que viven según los flujos globales del petróleo y sus crisis, otros que corresponden a empleados del sector público y, por último, grupos que no

\footnotetext{
${ }^{10}$ El golfo San Jorge es el nombre de la cuenca hidrocarburífera conformada por localidades que crecieron al calor de la extracción de petróleo y que actualmente está compuesto por las localidades de: Comodoro Rivadavia y Rada Tilly de la provincia de Chubut, y Caleta Olivia, Cañadón Seco, Pico Truncado y Las Heras de la provincia de Santa Cruz.

${ }^{11}$ Es una empresa del Estado argentino que desde la década de 1930 y hasta mediados de los 90 fue la única empresa encargada de la producción petrolera de Santa Cruz.
} 
encuentran inserción y llevan adelante reclamos por trabajo o bien son destinatarios de planes sociales ${ }^{12}$. Esos procesos de precarización laboral afectaron principalmente a los y las jóvenes, lo que ha dado surgimiento al problema de la "cuestión juvenil"13 (Salvia, 2008). Estudios recientes (Assusa, 2020) muestran que del período 2004 al 2019 en nuestro país se mantuvo una brecha que indica que los jóvenes (entre 15 y 24 años) están tres veces más expuestos al desempleo que los adultos (entre 25 y 65 años). Asimismo, allí se hace hincapié en que la informalidad laboral juvenil disminuyó 15 puntos, aunque ello ocurrió desigualmente entre jóvenes cuyas familias son del quintil con menos ingresos y jóvenes del quintil con más ingresos (a favor de estos últimos).

Focalizando en el escenario laboral de los jóvenes en Caleta Olivia, para 2010, entre los y las jóvenes de 18 a 25 años la tasa de desocupación alcanzaba el 34,6\% de la PEA en este grupo (Galaretto y Romero, 2010). En ese mismo estudio se menciona que, para una tasa de empleo del $48 \%$ del total de jóvenes, la rama de actividad donde desarrollan sus actividades laborales es en servicios sociales, comunales y personales, comercio y servicio a las empresas y construcción. Aquí, el trabajo en el petróleo o en servicios asociados a ello representa el 7\%. Un estudio reciente del Ministerio de Trabajo, Empleo y Seguridad Social de la Nación indica que, para 2017, en la provincia de Santa Cruz la tasa de desocupación en la PEA de jóvenes entre 18 y 24 años era de 25,1\%, de los cuales el 83,8\% era cesante (es decir, tenían empleo antes de ser desocupados). Con lo cual, el escenario laboral en Caleta Olivia se configura con entre un cuarto y un tercio de la población de jóvenes que busca trabajo que sufre la desocupación, de los cuales la gran mayoría ha devenido desempleada luego de tener trabajo. Asimismo, en una región cuya estructura económica y productiva sigue basándose en la extracción de petróleo, es mínimo el porcentaje de jóvenes que se inserta laboralmente en ese ámbito. Es desde este contexto sociolaboral que nos preguntamos por las vinculaciones entre la escuela y el mundo del trabajo desde el punto de vista de los y las

\footnotetext{
${ }^{12}$ También durante los años de dinamismo en la economía de la región, luego del período privatizador de fines del siglo XX, se han generado empleos formales en sectores de construcción, comercio y servicios (Rojo y Rotondo, 2009).

${ }^{13}$ Es decir, la instalación de la situación laboral vulnerable de los jóvenes como "problema público", expresado por proliferación de políticas públicas y programas orientados a promover la inclusión laboral de los jóvenes.
} 
jóvenes, es decir, cómo los y las estudiantes de Nivel Secundario conciben la formación con relación a la salida laboral.

Ocuparse de la formación para el trabajo desde la escuela requiere considerar el lugar que se da a los saberes en este escenario sociolaboral, así como los atributos de los trabajadores que se demandan. Como sostenía Gallart (1997), el carácter cambiante o volátil, asociado a la necesidad de respuestas rápidas propias de la producción a demanda en el modo de producción flexible, implican particulares exigencias de saberes a los trabajadores. Estos han sido conceptualizados bajo la noción de competencias, cuya particularidad es estar en estado de permanente cambio e involucrar la subjetividad del trabajador en la resolución de problemas en situaciones concretas de trabajo (Gallart, 1997; Jacinto y Milenaar, 2012). Ello conlleva ciertas tensiones en relación con los saberes necesarios para el trabajo y que deben ser brindados desde la formación escolar ya que "la competencia no proviene de la aprobación de un currículo escolar formal, sino de un ejercicio de aplicación de conocimientos en circunstancias críticas" (Gallart, 1997: 4). Ello ha implicado una ruptura con la secuencia formación-empleo que caracterizaba el pasaje de la escuela al trabajo en el modelo fordista-keynesiano (Dubar, 2001; Gallart, 1997; Jacinto, 2010). Es decir, en tiempos de capitalismo flexible, se reconoce que los sujetos deben insertarse muchas veces en el mercado de trabajo (Miranda y Corica, 2015; Miranda, 2017) y por lo tanto son llamados a "reciclar" sus saberes en general y sus saberes escolares en particular, a fin de volverse empleables $^{14}$ (Gallart, 1997; Jacinto y Milenaar, 2012).

Consideramos que tanto el concepto de competencias como el de empleabilidad son parte de las lógicas de formación dentro del diagrama de una gubernamentalidad neoliberal (Foucault, 2007). Es decir, la formación permanente, el aprender a aprender, el saber ser, entre otros, implican atributos y comportamientos que se buscan producir a fin de formar sujetos que sean "empresarios de sí mismos" (Foucault, 2007). Por lo tanto, en este esquema

\footnotetext{
${ }^{14}$ Siguiendo a Jacinto y Millenaar (2012), la noción de empleabilidad hace referencia al conjunto de disposiciones para el trabajo, competencias y saberes prácticos. Cabe remarcar que las políticas de empleo bajo la perspectiva de la empleabilidad en los 90 se orientaban a la enseñanza de habilidades técnicas, mientras que desde los 2000 hubo una reorientación de estas políticas hacia la formación de disposiciones actitudinales (Jacinto, 2010; Gutiérrez y Assusa, 2019).
} 
de la pedagogía de las competencias (Grinberg, 2008), la falta de empleo se transforma en un problema de sujetos (in)competentes y supone que la formación debe estar dirigida a que estos puedan y sepan invertir en sí mismos, tal como lo proponen las versiones clásicas y actuales del capital humano.

Ahora bien, en el estudio del vínculo de educación y trabajo se hace necesario diferenciar entre escuelas técnicas y bachilleratos, ya que ella refiere al carácter específico sobre el que se ha constituido históricamente el contenido de la educación secundaria: las escuelas técnicas han estado principalmente orientadas a la formación para el trabajo y las escuelas nacionales o comunes, centradas en una formación humanista (Gallart, 2006; Camilloni, 2006). En lo que respecta a la modalidad de educación técnica, las características de la reforma educativa de los 90, ajustadas a las demandas del mercado, se asentaron sobre una crítica, entre otras, a la especialización en la formación de técnicos ${ }^{15}$ (Gallart, 2006; Riquelme, 2006). Aún luego de la reforma de educación técnico-profesional a principios de este siglo ${ }^{16}$, hoy los egresados de las técnicas se enfrentan a requerimientos de saberes de las empresas en que priorizan atributos actitudinales (ganas de trabajar y aprender), antes que los saberes específicos del perfil profesional de formación técnica (Figari, 2017; Maturo, 2017). Al compás de estos procesos a nivel nacional, en Caleta Olivia la privatización de YPF implicó un desdibujamiento de las relaciones entre calificaciones y posiciones en la organización del trabajo de la empresa, en que los trabajadores con título técnico ocupaban el lugar de "jefes"17 (Alvarez, 2008). Siguiendo a Ruiz y Muñoz (2008), la reestructuración de la organización del trabajo en los 90, ligada a la multifuncionalidad y polivalencia, implicó una gran reducción del personal calificado y un proceso de subcontratación, el cual requería de trabajadores de menor calificación. De hecho, la informatización y robotización de procesos

\footnotetext{
${ }^{15}$ Según Gallart (2006), ello se expresó en la prolongación de la educación básica y la consiguiente reducción de años y horas curriculares para la formación técnica específica.

${ }^{16}$ Referimos a las reformas asociadas a la Ley de Educación Técnico Profesional N²6058 de 2005, que prioriza el aumento de la carga horaria de clases en el taller, incremento del financiamiento específico, homologación de títulos y certificados, promoción en compra de equipamiento y actualización tecnológica de las escuelas (Riquelme, 2006).

17 Según la autora, se distinguía los cargos de "jefes" (ocupados por personal con titulación de ingenieros y técnicos), de los "empleados" (trabajadores del sector administrativo) y de los "obreros" (quienes trabajaban en boca de pozo).
} 
productivos en la actividad petrolera en la región conllevan una demanda de trabajadores con cada vez mayor calificación, pero al mismo tiempo son los sectores con menores niveles de absorción de empleo (Schwaitzer, 2012; Márquez, 2017).

Situados en este marco de debates, nos preguntamos por las maneras en que los y las estudiantes de la escuela secundaria piensan las vinculaciones de su formación con relación al mundo del trabajo en Caleta Olivia. Para ello, a continuación se trabajará sobre las miradas que ellos y ellas otorgan a la escuela en general y con relación al mundo del trabajo en particular, teniendo en cuenta las condiciones socioeconómicas del emplazamiento escolar, la modalidad de la formación y sus diferentes momentos en el proceso de su escolaridad secundaria.

\section{Las miradas sobre lo escolar y la formación para el trabajo}

Pérez (2014) da cuenta de dinámicas de escolarización en que las instituciones ubicadas en territorios con bajos indicadores de pobreza se conforman de poblaciones escolares segmentadas, es decir de diferentes segmentos urbanos de la ciudad de Caleta Olivia, pero "para el caso de escuelas ubicadas en territorios con altos indicadores de pobreza, la población escolar se conforma de manera más fragmentada, es decir de alumnos concentrados alrededor del emplazamiento escolar" (Pérez, 2014: 108). Estas lógicas de fragmentación social y urbana (Veiga, 2009; Saraví, 2015) pueden estar presentes en las condiciones de escolarización desde el Nivel Primario y persisten para el Nivel Secundario, aunque profundizándose fundamentalmente por las características en este nivel de enseñanza. Ello contribuye a la producción de circuitos de escolarización que son formalmente iguales, pero en los que se producen procesos de formación desiguales (Saraví, 2015). De esta manera, nos proponemos indagar sobre las percepciones de los y las estudiantes de escuelas ubicadas en espacios urbanos con diferentes condiciones socioeconómicas. Así, en la Tabla $N^{\circ} 2$ se expresan los sentidos que los y las estudiantes dan sobre la importancia de concurrir a la escuela en función del emplazamiento urbano del establecimiento al que asisten. 
Tabla $\mathbf{N}^{\circ}$ 2. La importancia de concurrir a la escuela según los y las estudiantes, según NBI de radio escolar y año en curso. Caleta Olivia. En \% $(\mathrm{N}=150)$

\begin{tabular}{|l|r|r|r|r|r|r|}
\hline & \multicolumn{2}{|c|}{ NBI Bajo } & \multicolumn{2}{c|}{ NBI Medio } & \multicolumn{2}{c|}{ NBI Alto } \\
\cline { 2 - 7 } & $\begin{array}{c}\text { Primer } \\
\text { año }\end{array}$ & $\begin{array}{c}\text { Último } \\
\text { año }\end{array}$ & $\begin{array}{c}\text { Primer } \\
\text { año }\end{array}$ & $\begin{array}{c}\text { Último } \\
\text { año }\end{array}$ & $\begin{array}{c}\text { Primer } \\
\text { año }\end{array}$ & $\begin{array}{c}\text { Último } \\
\text { año }\end{array}$ \\
\hline Para el trabajo & $90,9 \%$ & $81,1 \%$ & $93,8 \%$ & $85,7 \%$ & $72,7 \%$ & $71,4 \%$ \\
\hline Para continuar estudios superiores & $45,5 \%$ & $70,3 \%$ & $56,3 \%$ & $64,3 \%$ & $42,4 \%$ & $64,3 \%$ \\
\hline Para ser un buen ciudadano & $54,5 \%$ & $54,1 \%$ & $50,0 \%$ & $57,1 \%$ & $42,4 \%$ & $28,6 \%$ \\
\hline Para ser una buena persona & $59,1 \%$ & $37,8 \%$ & $56,3 \%$ & $57,1 \%$ & $57,6 \%$ & $42,9 \%$ \\
\hline Es poco, pero algo hace por mí & $31,8 \%$ & $16,2 \%$ & $31,3 \%$ & $14,3 \%$ & $33,3 \%$ & $25,0 \%$ \\
\hline No es importante lo que enseña & $0 \%$ & $2,7 \%$ & $0 \%$ & $0 \%$ & $0 \%$ & $3,6 \%$ \\
\hline
\end{tabular}

Fuente: elaboración propia.

Una primera lectura de esta tabla indica que para la gran mayoría de los y las estudiantes, la escuela está asociada y es importante con relación al trabajo, sin importar el emplazamiento urbano. Es el motivo que para los y las estudiantes adquiere mayor fuerza (valores que van del 71 al 93\% de los casos) en comparación con las otras razones. Observamos que ello tiene mayor peso en el primer año en las escuelas de los diferentes NBI, y dichos valores disminuyen levemente hacia el último año en todos los casos. En contraste con ello, en la tabla se observan diferencias entre la importancia de la escuela para el trabajo y aquella que asignan al estudio. En primer lugar, el porcentaje de estudiantes que considera que la escuela es importante para continuar estudios en el primer año (valores del $42 \%$ al $56 \%$ ) es marcadamente inferior en comparación con la importancia asociada al trabajo. Asimismo, en todos los emplazamientos aumenta en el último año. Por lo tanto, si al ingresar al Nivel Secundario consideran que la escuela es importante principalmente para el trabajo, en el último año disminuye su valor, aunque sigue siendo una perspectiva central y se produce un incremento de la relevancia para los estudios superiores.

Estos sentidos se van relativizando y adquiriendo criterios de realidad a medida que la escolaridad transcurre, dado que comienzan a asociar directamente a aquello que ocurre con el trabajo y en tanto que, como mencionamos, el pasaje de la escuela secundaria al trabajo ha dejado de ser lineal (Jacinto, 2010). Como muestran diversos estudios, los jóvenes saben 
que el título secundario es condición necesaria pero no suficiente para acceder a un empleo (Jacinto, 2010; Corica, 2012). A ello se suma la obligatoriedad y masificación del nivel promovidas por las reformas de principio de este siglo que produjeron expectativas en las aspiraciones de los y las jóvenes asociadas a continuar estudios superiores. De aquí que el sentido propedéutico del Nivel Secundario adquiere relevancia y aumenta en los últimos años (Corica, 2012; Miranda y Corica, 2015). Si bien este incremento se da de manera general hay que considerar que, como señalan las investigaciones referenciadas, el sentido propedéutico y el sentido laboral de la formación escolar secundaria no se dan de la misma manera en todos los sectores sociales.

Siguiendo el emplazamiento de las escuelas, la disminución de la importancia asociada al trabajo entre el primer y el último año escolar se produce en proporción más fuerte en zonas con NBI Bajo y NBI Medio (90,9\% a 81,1\% y 93,8\% a 85,7\% respectivamente), mientras que es mínima en territorios con NBI Alto (72,7\% a 71,4\%). También observamos que el aumento de esos sentidos propedéuticos que se producen del primer al último año se da principalmente en zonas con NBI Bajo y NBI Alto (45,5\% a 70,3\% y 42,4\% a 64,3\%, respectivamente). Es decir, por un lado, en escuelas emplazadas en sectores con índices menores de pobreza, el aumento de la importancia de la escuela asociada a continuar estudios se produce junto con una disminución de la importancia relacionada con el trabajo. Mientras que, por otro lado, en las escuelas ubicadas en zonas con mayor pobreza la importancia en relación con el trabajo no se modifica sustancialmente al ingresar o finalizar el Nivel Secundario, pero sí aumenta aquella asociada a continuar estudios superiores. Siguiendo a Corica (2012), ello se relaciona con las expectativas laborales que suelen tener los y las jóvenes de estos sectores sociales: mientras que los jóvenes con menos urgencias económicas piensan en trayectorias de formación permanente y a más a largo plazo, así como en trabajos que les permitan cursar estudios superiores, los y las jóvenes de sectores en condición de mayor pobreza piensan en trabajos temporales que no requieren una formación específica, sino principalmente el título secundario. Por lo tanto, que la importancia de la escuela para el trabajo sea similar tanto en estudiantes ingresantes como en quienes ya están finalizando el Nivel Secundario en las escuelas en zonas de mayor pobreza, se puede relacionar con la 
relevancia del título secundario; mientras que el incremento de la importancia del sentido propedéutico se asocia al hecho que, sobre todo en estos sectores, seguir estudiando se transforma prácticamente en una obligación, así como en una "salida por arriba" (Langer y Esses, 2019).

Como hemos mencionado, se hace necesario diferenciar entre escuelas técnicas y bachilleratos, dado que históricamente las primeras han estado principalmente orientadas al trabajo. En Caleta Olivia, la formación técnica de Nivel Secundario surge orientada a la actividad petrolera, la cual, además, se pensaba con un carácter terminal, dada las expectativas de ingreso inmediato a YPF (Palma Godoy, 1999; Gutiérrez y Romero, 2013). Por ello, otra forma de analizar cómo se diferencian esas miradas de los y las estudiantes es a partir de considerarlas con relación a la modalidad técnica o bachillerato de la escuela a la que asisten, tal como se hace en la Tabla $N^{\circ} 3$.

Tabla $N^{\circ}$ 3. La importancia de concurrir a la escuela según los y las estudiantes de distintas modalidades y según año en curso. En \%. Caleta Olivia. $(\mathrm{N}=302)$

\begin{tabular}{|l|r|r|r|r|}
\hline \multirow{2}{*}{} & \multicolumn{2}{|c|}{ Técnicas } & \multicolumn{2}{c|}{ Bachilleratos } \\
\cline { 2 - 5 } & Primer año & Último año & Primer año & Último año \\
\hline Para el trabajo & 92,0 & 80,4 & 83,1 & 78,5 \\
\hline Para continuar estudios superiores & 60,0 & 78,4 & 46,5 & 67,1 \\
\hline Para ser un buen ciudadano & 44,0 & 50,0 & 47,9 & 45,6 \\
\hline Para ser una buena persona & 48,0 & 37,3 & 57,7 & 43 \\
\hline Es poco, pero algo hace por mí & 28,0 & 15,7 & 32,4 & 19 \\
\hline No es importante lo que enseña & 2,0 &, 0 &, 0 & 2,5 \\
\hline
\end{tabular}

Fuente: elaboración propia.

En sintonía con ese mandato fundacional de la escuela técnica en Caleta Olivia, los y las estudiantes de esta modalidad asocian la importancia de la escuela al trabajo (92\% en primer año y $80,4 \%$ en el último año) en mayor proporción que para continuar estudios superiores (60\% y $78,4 \%$, respectivamente). Pero esa proporción de quienes consideran la importancia para seguir estudiando en escuelas técnicas es aún mayor a la de los y las estudiantes de bachilleratos (46,5\% en primer año; $67,1 \%$ en el último año). Ya desde el primer año la mayoría de las y los estudiantes de escuelas técnicas ingresan con el deseo de continuar 
estudios superiores. En línea con resultados de investigaciones en otras regiones con economías petroleras (Garino, 2019), las demandas de cada vez mayor nivel de calificación de saberes técnicos para este sector de la producción generan una ruptura de la visión del secundario técnico como nivel terminal, dado que "el sector demanda cada vez más personal con calificaciones elevadas, discontinuando lo que sucedía en épocas anteriores, en las que ingresaban trabajadores sin título secundario o bien con esta credencial como máximo nivel educativo alcanzado" (Garino, 2019: 206).

Por otro lado, esas fuertes expectativas por continuar estudios superiores ya desde el ingreso en las escuelas técnicas también están relacionadas con las características de la población que asiste a esas instituciones. En efecto, como mencionamos inicialmente, se tratan de las escuelas de gestión pública más demandadas en la localidad y hasta no hace mucho su ingreso implicaba un fuerte mecanismo de selectividad (Gutiérrez y Romero, 2013) ${ }^{18}$. Ante el escenario de devaluación de credenciales (Miranda y Corica, 2015; Salvia, 2008) que hemos mencionado y el acceso al Nivel Secundario de nuevos grupos sociales, esos mecanismos de selección siguen existiendo, pero de formas solapadas en término de las búsquedas de las familias mejor posicionadas, ya no de titulaciones más elevadas (Bourdieu, 1988), sino de recorridos de mejor calidad y privilegio (Braslavsky, 1985). Así, tal como se observa en la Tabla $N^{\circ} 3$, en las expectativas de los y las estudiantes que logran ingresar a escuelas técnicas hay un marcado componente propedéutico.

Hasta aquí hemos discutido los sentidos que expresan los y las estudiantes en la relación entre educación y trabajo en general, más allá de la escuela a la que concurren. Ahora bien, cuando el interrogante remite a la escuela particular a la que asisten, hay diferentes tonalidades que se expresan más claramente según el NBI de emplazamiento escolar y la modalidad de la escuela que se van acentuando. La consideración del emplazamiento urbano de la escuela cobra mayor relevancia cuando los y las estudiantes elaboran una mirada que se sitúa espacialmente en la escuela en la que están, a diferencia de lo que expresan cuando reflexionan sobre la escuela en un sentido genérico, tal como expresa la

\footnotetext{
${ }^{18}$ Los autores indican que hasta 2012, si bien la inscripción era abierta, se confeccionaba una lista con orden de mérito según el rendimiento escolar en el Nivel Primario.
} 
Tabla No 4. Allí, la primera diferencia que expresa la relación entre la razón de asistencia a esa escuela y el emplazamiento de la institución es que el prestigio tiene mayor peso hacia zonas con NBI Bajo (45,5\% en el primer año y 45,9\% en el último año). Por contraste, para los y las estudiantes de las escuelas ubicadas en los NBI con mayor pobreza, es menor el porcentaje y además disminuye de primero al último año (18,8\% y 7,1\% en NBI Medio; 24,2\% y $3,6 \%$ en NBI Alto).

Tabla № 4. Las razones por la que asisten los y las estudiantes a su escuela, según NBI de emplazamiento escolar y año en curso. En \%. Caleta Olivia. $(\mathrm{N}=\mathbf{2 4 5})$

\begin{tabular}{|l|r|r|r|r|r|r|}
\hline \multirow{2}{*}{} & \multicolumn{2}{|c|}{ NBI Bajo } & \multicolumn{2}{c|}{ NBI Medio } & \multicolumn{2}{c|}{ NBI Alto } \\
\cline { 2 - 7 } & $\begin{array}{c}\text { Primer } \\
\text { año }\end{array}$ & $\begin{array}{c}\text { Último } \\
\text { año }\end{array}$ & $\begin{array}{c}\text { Primer } \\
\text { año }\end{array}$ & $\begin{array}{c}\text { Último } \\
\text { año }\end{array}$ & $\begin{array}{c}\text { Primer } \\
\text { año }\end{array}$ & $\begin{array}{c}\text { Último } \\
\text { año }\end{array}$ \\
\hline Es prestigiosa & $45,5 \%$ & $45,9 \%$ & $18,8 \%$ & $7,1 \%$ & $24,2 \%$ & $3,6 \%$ \\
\hline $\begin{array}{l}\text { Se relaciona con lo que quiero } \\
\text { trabajar }\end{array}$ & $27,3 \%$ & $37,8 \%$ & $25,0 \%$ & $14,3 \%$ & $24,2 \%$ & $17,9 \%$ \\
\hline $\begin{array}{l}\text { Queda cerca de mi casa } \\
\text { La orientación me interesa }\end{array}$ & $18,2 \%$ & $32,4 \%$ & $50,0 \%$ & $28,6 \%$ & $69,7 \%$ & $57,1 \%$ \\
\hline Hay buen ambiente social y cultural & $56,4 \%$ & $51,4 \%$ & $6,3 \%$ & $35,7 \%$ & $12,1 \%$ & $14,3 \%$ \\
\hline Conviene el horario & $13,6 \%$ & $27,8 \%$ & $37,5 \%$ & $7,1 \%$ & $27,3 \%$ & $28,6 \%$ \\
\hline Mis hermanos van allí & $27,3 \%$ & $16,2 \%$ & $18,8 \%$ & $7,1 \%$ & $42,4 \%$ & $28,6 \%$ \\
\hline No hay movilidad para ir más lejos & $9,1 \%$ & $8,1 \%$ & $18,8 \%$ & $7,1 \%$ & $33,3 \%$ & $10,7 \%$ \\
\hline Es más fácil terminar acá & $9,1 \%$ & $21,6 \%$ & $31,3 \%$ & $14,3 \%$ & $15,2 \%$ & $32,1 \%$ \\
\hline No había lugar en la que quería & $9,1 \%$ & $18,9 \%$ & $50,0 \%$ & $35,7 \%$ & $24,2 \%$ & $28,6 \%$ \\
\hline Aceptan repitentes & $27,3 \%$ & $8,1 \%$ & $25,0 \%$ & $7,1 \%$ & $27,3 \%$ & $14,3 \%$ \\
\hline No hay chicos con problemas & $13,6 \%$ & $8,1 \%$ & $6,3 \%$ &, $0 \%$ & $12,1 \%$ & $10,7 \%$ \\
\hline
\end{tabular}

Fuente: elaboración propia.

En esos sentidos diferenciales que tienen sobre el prestigio de las escuelas están presentes, entre otras dimensiones, los efectos del "estigma territorial" (Waquant, 2001) que recae sobre los contextos de pobreza. De hecho, en el imaginario social de la localidad, cuando se habla de las escuelas ubicadas en NBI Medio y NBI Alto, suele identificárselas a partir del barrio en que se emplazan (Langer, Cestare y Martincic, 2017), a diferencia de aquello que sucede con las ubicadas en territorios con NBI Bajo que se las emparenta por su número o nombre. 
Por otro lado, los y las estudiantes de escuelas ubicadas en los barrios con mayor pobreza señalan que la cercanía de la escuela con respecto al hogar es el principal motivo por el que concurren allí (69,7\% en primer año y $57,1 \%$ en último año en NBI Alto). Aquí hay que considerar no solo las condiciones de pobreza del radio censal de esas escuelas, sino también la ubicación espacial en el tejido urbano. En el Mapa 1, presentado al comienzo, se observa que las escuelas en zonas de mayor pobreza están emplazadas en zonas periféricas de la ciudad. La accesibilidad a la escuela es algo que los y las estudiantes valoran y que se vuelve clave para estos espacios de la urbe (Pérez, 2014; Grinberg, Pérez y Venturini, 2013; Cestare, Langer y Villagrán, 2015).

En cuanto a la asistencia a la escuela porque se relaciona con el trabajo, se observa que en todos los NBI esa razón ocupa del cuarto al quinto lugar. Ello se diferencia de lo expresado sobre la importancia de la escuela en general, donde la utilidad para el trabajo ocupaba el primer lugar. Si, por un lado, los y las estudiantes consideran que la escuela es importante principalmente para el trabajo, al mismo tiempo señalan que la razón de asistencia a sus escuelas en particular no tiene tanta relación con el trabajo. Ahora bien, vemos que de primer a último año esos motivos asociados al trabajo cambian de distinta manera en cada NBI. Mientras en NBI Bajo, el porcentaje aumenta en estudiantes que están en último año (de $27,3 \%$ a $37,8 \%$ ), en NBI Medio y NBI Alto los valores porcentuales disminuyen (de $25 \%$ a $14,3 \%$ y de $24,2 \%$ a $17,9 \%$, respectivamente). En suma, si entre estudiantes que ingresan al Nivel Secundario en zonas con diferentes NBI hay semejanzas en cuanto a las razones de asistencia asociadas al trabajo, observamos que al finalizar la secundaria es donde se producen con mayor fuerza las diferenciaciones entre estudiantes de escuelas en esos distintos emplazamientos urbanos.

Es en esos recorridos diferenciales de las escolaridades de los y las estudiantes que se ponen en juego las dinámicas que producen circuitos educacionales diversos y desiguales, asociados a procesos de fragmentación escolar y urbana (Saraví, 2015; Pérez, 2014; Grinberg, Pérez y Venturini, 2013). Entendemos que estos circuitos diferenciales de escolarización se constituyen principalmente sobre la base del contenido y las dinámicas de la formación. Ello porque las orientaciones que se ofrecen en las escuelas ubicadas en los emplazamientos con 
mayor NBI son las que menos se relacionan con los sectores más dinámicos del mercado laboral en la región del golfo San Jorge ${ }^{19}$. En efecto, se observa en la Tabla 4 que el interés por la orientación es mayor en zonas con NBI Bajo tanto en primero como en último año (36,4\% y $51,4 \%$, respectivamente), en comparación con el peso que tiene en espacios con NBI Medio (6,3\% en primer año y 35,7\% en último año) y NBI Alto $(12,1 \%$ y $14,3 \%$, respectivamente). Por lo tanto, estos procesos contribuyen a diversificar y producir desigualdades en el proceso de formación de Nivel Secundario y operan en las consideraciones sobre la relación entre escuela y trabajo, las cuales tienden a diferenciarse sobre todo cuando piensan en sus escuelas en particular y se profundizan aún más hacia el último año.

A continuación, abordamos estas diferenciaciones recuperando las visiones de estudiantes ahora desde el punto de vista de la modalidad que cursan, en torno a esta misma idea de la razón por la que asisten, tal como se expresa en la Tabla Nº 5.

Tabla $\mathbf{N}^{\circ}$ 5. Las razones por la que asisten estudiantes a su escuela según modalidad y año en curso. En \%. Caleta Olivia. ( $N=302)$

\begin{tabular}{|l|r|r|r|r|}
\hline \multirow{2}{*}{} & \multicolumn{2}{|c|}{ Técnicas } & \multicolumn{2}{c|}{ Bachilleratos } \\
\cline { 2 - 4 } & Primer año & Último año & Primer año & Último año \\
\hline Es prestigiosa & $60,0 \%$ & $61,8 \%$ & $29,6 \%$ & 24,1 \\
\hline Se relaciona con lo que quiero trabajar & $52,0 \%$ & $58,8 \%$ & $25,4 \%$ & 26,6 \\
\hline Queda cerca de mi casa & $26,0 \%$ & $11,8 \%$ & $49,3 \%$ & 40,5 \\
\hline La orientación me interesa & $62,0 \%$ & $71,6 \%$ & $18,3 \%$ & 35,4 \\
\hline Hay buen ambiente social y cultural & $52,0 \%$ & $27,5 \%$ & $36,6 \%$ & 29,1 \\
\hline Conviene el horario & $24,0 \%$ & $1,0 \%$ & $28,2 \%$ & 24,1 \\
\hline Mis hermanos van allí & $24,0 \%$ & $17,6 \%$ & $25,4 \%$ & 12,7 \\
\hline No hay movilidad para ir más lejos & $4,0 \%$ & $2,9 \%$ & $14,1 \%$ & 16,5 \\
\hline Es más fácil terminar acá & $14,0 \%$ & $2,9 \%$ & $21,1 \%$ & 22,8 \\
\hline No había lugar en la que quería & $10,0 \%$ & $5,9 \%$ & $25,4 \%$ & 25,3 \\
\hline Aceptan repitentes & $20,0 \%$ & $2,9 \%$ & $26,8 \%$ & 10,1 \\
\hline No hay chicos con problemas & $24,0 \%$ & $9,8 \%$ & $11,3 \%$ & 7,6 \\
\hline
\end{tabular}

${ }^{19}$ Un análisis sobre la relación entre distribución de oferta de orientaciones escolares, emplazamiento urbano de las escuelas y características del mercado laboral en la región del golfo San Jorge se encuentra en Guzmán, Langer y Grinberg (2020). 
Fuente: elaboración propia.

En la Tabla No 5 observamos que los motivos de asistir a una escuela de modalidad técnica se asocian principalmente al prestigio (60\% en primer año y $61,8 \%$ en último año), la relación con el trabajo (52\% y 58,8\%, respectivamente) y la orientación (62\% y 71,6\%, respectivamente). Prestigio, relación con el trabajo y la orientación son tres razones que expresan parte de esas fuertes elecciones iniciales que han hecho las familias y los y las estudiantes para llegar a este tipo de escuelas y por tanto, también, sus expectativas hacia el futuro. La escolaridad y la orientación de la institución vienen a confirmar parte de esas elecciones y expectativas futuras. En cambio, esas razones para quienes asisten a bachilleratos pierden peso y se destaca la cercanía con el hogar como principal motivo (49,3\% y 40,5\%, respectivamente). Aquí se profundizan las diferencias que ya expresaban los y las estudiantes de ambas modalidades en cuanto a la importancia de la escuela para el trabajo. Las particularidades de la formación en escuelas técnicas basadas en el aprendizaje situado en espacios de taller y laboratorio, la orientación con un fuerte sentido de especialización y las pasantías en tanto experiencias en situaciones reales de trabajo (Gallart, 2006) contribuyen a la fuerte relación con el trabajo y la orientación escolar que están presentes en las razones de asistencia escolar de estudiantes que cursan en esta modalidad. En cambio, en los bachilleratos no solo que ello no existe, sino que esas elecciones iniciales en función de la cercanía es lo que va marcando el rumbo de cómo viven sus escolaridades allí. Específicamente, con relación a la modalidad, se observa una persistencia de la diferenciación histórica que en Caleta Olivia ha marcado una relación más estrecha con el trabajo para la formación secundaria técnica a diferencia de lo ocurrido en bachilleratos. Al interior de estos, ese decrecimiento de las razones asociadas al trabajo para asistir a sus escuelas se profundiza aún más en los y las estudiantes de instituciones ubicadas en zonas con más NBI.

En definitiva, las miradas de los y las estudiantes sobre la relación entre escuela y trabajo se producen en un contexto local donde la historia de la ciudad, la vida cotidiana, así como las dinámicas sociolaborales están fuertemente condicionadas por la actividad petrolera. Pero 
pese a ello, los y las jóvenes que logran insertarse en el mundo del trabajo lo hacen principalmente en el sector servicios, comercio y construcción. Como hemos señalado, es mínimo el porcentaje de jóvenes que ingresan al ámbito laboral del petróleo ${ }^{20}$. Es en este escenario que esas perspectivas que relacionan escuela y trabajo se producen diferencial y desigualmente según la modalidad de la escuela, así como también según el año escolar y el emplazamiento urbano de las instituciones.

\section{Reflexiones finales}

En este artículo se propuso describir las características que asume la formación para el trabajo desde la perspectiva de estudiantes de escuelas secundarias de la localidad de Caleta Olivia, atendiendo a la importancia que otorgan a la educación secundaria con relación al trabajo. Esas lecturas estuvieron atravesadas por las características que asumen el trabajo y los saberes para el trabajo en el capitalismo flexible (Harvey, 2000; Gallart, 1997), y sus particularidades en la región petrolera del golfo San Jorge. Específicamente en Caleta Olivia, en los últimos años la mayoría de jóvenes que se inserta, lo hace en el comercio, construcción o servicio, y es mínimo el porcentaje de jóvenes que ingresa en el sector petrolero. Asimismo, la gran mayoría de los y las jóvenes que se encuentran desocupados lo han hecho luego de tener trabajo. Es decir, la importancia de la escuela y las razones de asistir a sus escuelas, en particular de los y las estudiantes, se produce en un escenario laboral local que presenta las características inciertas y fluctuantes propias del empleo y el trabajo de los últimos años.

Estas miradas estudiantiles fueron analizadas vinculando la dinámica de los procesos laborales con el sistema escolar, a la luz de las transformaciones de la tradicional lógica de las redes de escolarización (Baudelot y Establet, 1975), que forma y distribuye diferencialmente a los sujetos según las distintas posiciones del mercado de trabajo y la estructura social. En este sentido, la pregunta abarcó la relación con la fragmentación urbana (Veiga, 2009; Saraví, 2015) y la forma en que el sistema educativo de Nivel Secundario se

\footnotetext{
${ }^{20} \mathrm{Y}$ quienes lo hacen se incorporan principalmente en empresas de servicios al petróleo (Garino, 2019).
} 
constituye sobre la diferenciación de la enseñanza técnica o bachillerato (Gallart, 2006; Camilloni, 2006). Ello se hizo a través de la descripción de las perspectivas de los y las estudiantes, prestando atención a las condiciones de pobreza del emplazamiento escolar, a la modalidad de las escuelas y al año escolar que cursan.

Algunos de los principales resultados obtenidos remiten a que para los y las estudiantes que asisten a escuelas de todos los NBI, el trabajo es el fundamento principal a la hora de pensar la importancia de la escuela. Sin embargo, esa importancia de la escuela asociada al trabajo disminuye en gran proporción en estudiantes del último año comparativamente a aquellos que inician, al mismo tiempo que aumenta la consideración para seguir estudiando de aquellos que están terminando el nivel. Ello ocurre en las miradas de quienes asisten a escuelas en zonas con distintos NBI, aunque con mayor fuerza en aquellos que concurren en espacios con menor pobreza. Dichas mutaciones, en las perspectivas de los y las estudiantes durante el proceso de escolarización, refieren a un reconocimiento de la insuficiencia del título secundario y las necesidades de seguir estudiando ante las demandas de un trabajador polivalente y la relevancia de la formación continua en el mundo del trabajo hoy.

Por otro lado, los sentidos de los y las jóvenes expresan una diferenciación más marcada cuando piensan acerca de su escuela en particular y atendiendo a dos variables distintas. La primera tiene que ver con la relación entre la configuración urbana y la desigualdad en donde el prestigio de la escuela es el motivo principal para quienes asisten a las escuelas ubicadas en NBI Bajo, mientras que la cercanía de la escuela con respecto al hogar de residencia es el fundamento con mayor peso para quienes concurren a instituciones ubicadas en NBI Medio y NBI Alto. Ello indica una tendencia que se mantiene de investigaciones anteriores en la localidad, las cuales muestran que estas características no son exclusivas del Nivel Secundario, sino que están presentes ya desde el nivel primario de escolarización (Cestare, Langer y Villagrán, 2015; Grinberg, Pérez y Venturini, 2013; Pérez, 2014). Por otro lado, se observó que el trabajo y la orientación son las razones principales de asistencia escolar que esgrimen los y las estudiantes cuando se trata de las escuelas técnicas a diferencia de los y las que concurren a bachilleratos. 
En síntesis, podemos señalar que en un escenario sociolaboral local en que se exigen cada vez más calificaciones y tiene cada vez menos vacantes en los lugares más estables del mercado de trabajo (Márquez, 2017), los y las estudiantes de escuelas públicas expresan sentidos diversos y desiguales entre sí. En efecto, la modalidad de la escuela es uno de los elementos centrales en esas diferencias entre las perspectivas de los y las estudiantes. El prestigio de la institución y la relación más estrecha con el mundo del trabajo están más presentes en estudiantes de las escuelas técnicas. Asimismo, entre bachilleratos el emplazamiento urbano de las instituciones parece indicar un segundo elemento diferenciador y de desigualdad en esta modalidad. Allí los y las estudiantes que asisten a escuelas secundarias en zonas con NBI Alto son quienes expresan mayores distancias con respecto del prestigio de sus escuelas y la vinculación con el trabajo.

Aun así, consideramos que las razones asociadas al trabajo, los estudios propedéuticos, el prestigio, la orientación y la cercanía se combinan de múltiples formas en los y las estudiantes con relación al emplazamiento socioeconómico, el año en curso y las modalidades que cursan. No se pueden asignar lugares, posiciones ni disposiciones a unos ni a otros. Como mencionamos, las trayectorias postsecundaria han dejado de ser lineales y en los procesos de estructuración social las maneras de resignificación de la experiencia escolar tienden a diversificarse cada vez más (Jacinto, 2013; Miranda, 2017). Es decir, el entramado que se va construyendo a través de estas miradas es mucho más complejo que en los procesos de segmentación escolar que describían los clásicos de la sociología de la educación de los 60 y los 70. Ni siquiera es posible vislumbrar del todo esas lógicas de la fragmentación escolar que en los 90 y principios del siglo XXI se han descripto. De forma tal que mientras vivimos tiempos en que la escuela parece encontrar una condición clave de su existencia en la formación para el trabajo cuando nos acercamos a las dinámicas de la escolaridad, esas oportunidades están diferencial y desigualmente distribuidas.

\section{Bibliografía}


Álvarez, M. (2008). YPF en la Región Austral: Saberes, trabajo y perfiles laborales. En Ruiz, J.D. (coord.) Petróleo y región austral: Saberes del trabajo y educación técnica. Reconfiguraciones y nuevas subjetividades (pp. 119-137). Buenos Aires: Dunken.

Antunes, R. (2009). Diez tesis sobre el trabajo del presente (y el futuro del trabajo). En Neffa, J.C.; De la Garza Toledo, E. y Muñiz Terra, L. (comps.) Trabajo, empleo, calificaciones profesionales, relaciones de trabajo e identidades laborales. Vol. I, Buenos Aires: Clacso.

Assusa, G. (2020). Jóvenes vulnerados e invisibilizados: Desigualdad y juventud en la Argentina de los últimos 15 años. En Dossier de Publicaciones universitarias en derechos humanos. Universidad Nacional de Córdoba.

Baudelot, C. y Establet, R. (1975). La escuela capitalista. Madrid: Siglo XXI Editores.

Bellet, C. y Llop, J. (2004) Miradas a otros espacios urbanos: las ciudades intermedias. Scripta Nova. Revista Electrónica de Geografía y Ciencias Sociales, VIII, 165. Disponible en: http://www.ub.edu/geocrit/sn/sn-165.htm

Bourdieu, P. (1988). La distinción. Criterio y bases sociales del buen gusto. Madrid: Taurus.

Braslavsky, C. (1985). La discriminación educativa en Argentina. Buenos Aires: Flacso-Grupo Editor Latinoamericano.

Camilloni, A. (2006). El saber sobre el trabajo en el currículo escolar. Anales de la educación común, (3)2, 112-117.

Castel, R. (1997). La metamorfosis de la cuestión social. Una crónica del salariado. Buenos Aires: Paidós.

Cestare, M.; Langer, E. y Villagrán, C. (2015). Estrategias y acciones en una escuela a la que concurren adolescentes y jóvenes de sectores populares en Caleta Olivia/Santa Cruz. Novedades Educativas. Escuela secundaria: nuevos sentidos, 294, 10-15.

Corica, A. (2012). Las expectativas sobre el futuro educativo y laboral de jóvenes de la escuela secundaria: entre lo posible y lo deseable. Última década, 36, 71-95.

De Ibarrola, M. (2016). Claroscuros en las relaciones entre la escolaridad y el trabajo. Configuraciones y límites. Revista Páginas de Educación, (2)9.

De la Garza, E. (coord). (2000). Tratado latinoamericano de sociologia del trabajo. México: El Colegio de México, Facultad Latinoamericana de Ciencias Sociales, Universidad Autónoma Metropolitana, Fondo de Cultura Económica.

Deleuze, G. (2006). Post-scriptum sobre las sociedades de control. Polis, 13. Disponible en: http://journals.openedition.org/polis/5509

Dubar, C. (2001). La construction sociale de l'insertion professionnelle. Education et sociétés, 7, 23-36.

Feres, J.C. y Mancero, X. (2001). El método de las necesidades básicas insatisfechas (NBI) y sus aplicaciones en América Latina. Santiago de Chile: Cepal. 
Figari, C. (2017). La trama del capital: La hegemonía empresaria en Argentina. Buenos Aires: Editorial Biblos.

Figari, C. y Palermo, H. (2010). Producción y reproducción de sentidos en un enclave petrolero. La privatización de YPF en Comodoro Rivadavia. Revista Trabajo, (6)4,146165.

Foucault, M. (2007). Nacimiento de la biopolítica: curso en el Collège de France: 1978-1979. Buenos Aires: Fondo de Cultura Económica.

Galaretto, M. y Romero, J. (2010). La educación de los jóvenes de la región en el nuevo escenario productivo. $10^{\circ}$ Congreso Nacional de Estudios del Trabajo (paper).

Gallart, M. (1997). Los cambios en la relación escuela-mundo laboral. Revista Iberoamericana de Educación, 15, 1-11.

Gallart, M. (2006). La escuela técnica industrial en Argentina: ¿un modelo para armar? Montevideo: Cinterfor-OIT.

Garino, D. (2019). El mundo del petróleo y del gas en Vaca Muerta. RevIISE, 13, 193-208.

Grinberg, S. (2008). Educación y poder en el Siglo XXI. Gubernamentalidad y Pedagogía en las sociedades de gerenciamiento. Buenos Aires: Miño y Dávila.

Grinberg, S.; Pérez, A. y Venturini, M.E. (2013). Configuraciones urbanas y escolares: notas de fragmentación educativa y territorial. En Grinberg, S. (coord.) La escuela not dead. Dispositivos pedagógicos, territorios y desigualdad (pp. 43-66). Río Gallegos: Ediciones Universidad Nacional de la Patagonia Austral.

Gutiérrez, A. y Assusa, G. (2019). Estrategias de inserción laboral y capital social. Un estudio sobre jóvenes de clases populares en Córdoba, Argentina. Última Década, 51.

Gutiérrez, E. y Romero, J. (2013). Política educativa: Una perspectiva crítica de las condiciones reales. ICT-UNPA, 71, 22-55.

Guzmán, M.; Langer, E. y Grinberg, S. (2020). Educación, trabajo y redes de escolarización en el capitalismo flexible: Un estudio en localidades del Golfo San Jorge. Revista Trabajo y Sociedad, (XXI)35, 603-623.

Harvey, D. (2000). La condición de la posmodernidad. Investigación sobre los orígenes del cambio cultural. Buenos Aires: Amorrortu.

Jacinto, C. (comp.) (2010). La construcción social de las trayectorias laborales de jóvenes. Políticas, instituciones, dispositivos y subjetividades (pp. 15-49). Buenos Aires: Teseo/IDES.

(2013). La formación para el trabajo en la escuela secundaria como reflexión crítica y como recurso. Propuesta Educativa, (40)22, 48-63.

Jacinto, C. y Millenaar, V. (2012). Los nuevos saberes para la inserción laboral. Formación para el trabajo con jóvenes vulnerables en Argentina. Revista Mexicana de Investigación Educativa, (52)17, 141-166. 
Langer, E y Esses, J. (2019). La salida es por arriba. Una historia de juventud, pobreza y educación. Buenos Aires: Grupo Editor Universitario.

Langer, E.; Cestare, M. y Martincic, H. (2017). Pobreza, educación, estigmatización y luchas por los derechos sociales. En el marco del XIX Congreso REDCOM Federalizar la comunicación: experiencias, utopías y recorridos pendientes. Comodoro Rivadavia (paper).

Márquez, D. (1995). Crisis socio-laboral y políticas de empleo: Algunas reflexiones para el análisis del caso comodorense. En Márquez, D. y Palma Godoy, M. (eds.) Distinguir y comprender: aportes para pensar la sociedad y la cultura en Patagonia (pp. 169-194). Comodoro Rivadavia: Edición Proyecciones patagónicas.

(2017). Del petróleo estatal al petróleo privado: continuidades y rupturas en el mundo sociolaboral de los trabajadores petroleros de la Cuenca del Golfo San Jorge durante las últimas tres décadas. En el marco de las XVI Jornadas Interescuelas. Universidad de Mar del Plata, Mar del Plata (paper).

Maturo, Y. (2017). Lo que ellas quieren. Requerimiento de las empresas respecto a las capacidades profesionales de los alumnos pasantes de la escuela técnica. En el marco del 13 Congreso Nacional de Estudios del Trabajo. Buenos Aires (paper).

Miranda, A. (2017). Presentación del Dossier Educación y Trabajo. Revista Latinoamericana de Estudios del Trabajo, (21)36, 95-105.

Miranda, A. y Arancibia, M. (2017). El futuro está incompleto: La construcción de trayectorias laborales sobre principios de siglo 21. Revista Trabajo y Sociedad, 28, 195-217.

Miranda, A. y Corica, A. (2015). Las actividades laborales y extraescolares de jóvenes de la escuela secundaria en la Argentina de principios del siglo XXI. Perfiles Educativos, (148)XXXVII, 100-118.

Palma Godoy, M. (1999). Antropología de la vida privada en Caleta Olivia. La construcción de la espacialidad juvenil como proyecto inconcluso. Trabajo presentado en la Maestría en antropología social, UNAM, Misiones (inédito).

Pérez, A. (2014). Políticas de escolarización, territorio y desigualdad educativa en épocas de gerenciamiento. Una mirada en clave territorial de la escolarización obligatoria estatal en el decenio de 2001-11. El caso de Caleta Olivia, provincia de Santa Cruz, Argentina. Universidad Nacional de Luján, tesis de Maestría.

Prevot Schapira, M.F. (2001). Fragmentación espacial y social: conceptos y realidades. Revista Perfiles Latinoamericanos, 019, 33-56.

Riquelme, G. (2006). La relación entre educación y trabajo: continuidad, rupturas y desafíos. En Anales de la educación común, 5, 2. 
Rojo, S. y Rotondo, S. (2009). Perfil de especialización del empleo registrado en el Golfo San Jorge: un diagnóstico desde la perspectiva del desarrollo local. Actas de $9^{\circ}$ Congreso Nacional de Estudios del Trabajo, Buenos Aires.

Ruiz, J. D. y Muñoz, N. (2008). La privatización de YPF y su repercusión en las subjetividades. En Ruiz, J.D. (coord.) Petróleo y región austral: Saberes del trabajo y educación técnica. Reconfiguraciones y nuevas subjetividades (pp. 91-118). Buenos Aires: Editorial Dunken.

Salvia, A. (1997). Crisis y reestructuración de complejos mineros: estudios de dos sistemas regionales patagónicos. En Salvia, A. y Panaia, M. (comps.) La Patagonia privatizada (pp. 36-48). Buenos Aires: Colección CEA-CBC.

(2008): Jóvenes Promesas. Trabajo, Educación y Exclusión Social en jóvenes pobres de la Argentina. Buenos Aires: Miño y Dávila.

Saraví, G. (2015) Juventudes fragmentadas. Socialización, clase y cultura en la construcción de la desigualdad. México: Flacso.

Schweitzer, A. (2012). Petróleo y territorios en la Provincia de Santa Cruz. Aproximaciones al estudio del espacio del Golfo de San Jorge. En Álvarez, M.; Michniuk, N. y Villanueva, M. (coords.) Educación y trabajo, miradas desde lo regional. Territorio y desigualdad de oportunidades (pp. 21-54). Buenos Aires: Editorial El Colectivo.

Sennett, R. (2000). La corrosión del carácter. Las consecuencias personales del trabajo en el nuevo capitalismo. Barcelona: Anagrama.

Veiga, D (2009). Desigualdades sociales y fragmentación urbana. En Poggiese, H. y Cohen Egler, T. (comps.) Otro desarrollo urbano: ciudad incluyente, justicia social y gestión democrática (pp. 51-61). Buenos Aires: Clacso.

Wacquant, L. (2001). Parias Urbanos. Marginalidad en la ciudad a comienzos del milenio. Buenos Aires: Manantial. 